\title{
Value of urine microscopy in predicting histological changes in the kidney: double blind comparison
}

\author{
AKOS Z GYÖRY, C HADFIELD, C S LAUER
}

\begin{abstract}
Fresh, first morning specimens of urine from 22 consecutive patients were examined by quantitative microscopy on the morning of renal biopsy; the renal biopsy samples were evaluated "blindly." Five patients showed no abnormality in the biopsy samples but eight had minimal, one mild, six moderate, and two severe histological changes. Comparison of the results of quantitative microscopy of urine with the presence or absence of histological evidence of disease showed that sensitivity was $88 \%$, specificity $83 \%$, accuracy $86 \%$, positive predictive value $93 \%$, and negative predictive value $71 \%$. When combined with microscopy of a second urine specimen these values were $100 \%, 50 \%, 87 \%, 85 \%$, and $100 \%$ respectively. There was a significant relation between number of casts and severity of the histological changes $(p<0.01)$. Comparison of renal functional abnormalities with histological findings gave values of $64 \%, 100 \%, 73 \%, 100 \%$, and $50 \%$ respectively.

It is concluded that quantitative microscopy of the first morning specimen of urine is a sensitive test with high predictive value for the presence or absence of renal disease. If no casts are detected in two early morning specimens the likelihood of finding anything more than minimal changes in a biopsy sample is virtually zero.
\end{abstract}

\section{Introduction}

In a previous study we showed that abnormalities of urinary sediment correlated with renal functional abnormalities, but

University Department of Medicine and Department of Anatomical Pathology, Royal North Shore Hospital of Sydney, St Leonards, Australia

AKOS Z GYÖRY, MD, FRACP, associate professor of medicine

C HADFIELD, BSC (med), $M B$, registrar

C S LAUER, MB, FRCPA, pathologist

Correspondence and requests for reprints to: Dr Akos Z Györy, Department of Medicine, Royal North Shore Hospital, St Leonards, 2065, Australia. only when microscopy was by a spot quantitative method on first morning urine specimens after centrifugation. ${ }^{1}$ The high power field method after centrifugation was quite inadequate.

In that study, which was retrospective and not coded, four patients were found to have abnormalities on renal biopsy as well as on quantitative microscopy. It was therefore decided to determine how well the results of renal biopsy and urine microscopy correlated and in particular to determine the sensitivity of urine microscopy in reflecting relatively minor histological abnormalities. This was thought especially important since reports of "silent" glomerulonephritis, especially in lupus, ${ }^{23}$ are still being reported, often leading to renal biopsy which might not on other grounds be necessary. Severe renal disease is usually reflected in abnormal blood biochemical values, but minor changes may well be accompanied by entirely normal renal function.

Our aim was therefore to compare prospectively the sensitivity, specificity, accuracy, and positive and negative predictive powers of quantitative urine microscopy of a single specimen in detecting the presence or absence of renal disease.

Urine specimens were handled routinely, as we wished to test a routine and not a research method in detecting renal disease. For the same reason we did not specifically include tests for reproducibility of the technique.

\section{Methods}

Fresh, first morning midstream specimens of urine were examined within one hour from 22 consecutive patients undergoing renal biopsy that day. Patients with orthostatic proteinuria were excluded. The biopsies were performed by another team for reasons unknown to us. The patients were not fasted overnight. Specimens of urine from each patient and a control (another patient with known renal disease) were code labelled by the ward sister and centrifuged; 9.5 of each $10 \mathrm{ml}$ was discarded and the resuspended samples examined and counted inside the marked area of a Fuchs-Rosenthal counting chamber, as described. ${ }^{1}$ In this paper "casts" refers to all types of casts; most, however, were granular and only one patient had casts that were exclusively hyaline (see below). Since we know of no adequate published data on which types of casts are pathological and which are not we regarded all as abnormal. Urine containing fewer than $15 \times 10^{3}$ casts (one per whole field), $3 \times 10^{6}$ white cells, and $1 \times 10^{6}$ red cells per litre was considered normal. ${ }^{4}$ The numbers, of casts were graded 
for comparison with severity of the histological abnormality as: $1=15 \times 10^{3}, 2=15-100 \times 10^{3}, 3=100-500 \times 10^{3}$, and $4=>500 \times 10^{3}$ casts per litre of urine.

Renal biopsy samples were examined first by the routine laboratory (not "blind") by light, immunofluorescence, and electronmicroscopical techniques where possible. Subsequently, when the study closed the specimens were again examined but by a pathologist not concerned in the original evaluation (CSL) and without knowledge of the patients' histories or diagnoses ("blind"). Light microscopical histological grading for comparison with the numbers of casts excreted was: normal $=0$; minimal change $=1$ (average number of glomerular nuclei less than 200, mesangium inapparent or present

TABLE I-Correlation of urinary casts with renal disease (see table II for raw data)

\begin{tabular}{lcc}
\hline & $\begin{array}{c}\text { "One look"* } \\
\left({ }_{0}\right)\end{array}$ & $\begin{array}{c}\text { "Two looks"* } \\
\left(0_{0}\right)\end{array}$ \\
\hline Sensitivity [TP/(TP + FN)] & $82(88)+$ & $100(100) \dagger$ \\
Specificity [TN/(FP + TN)] & $80(83)$ & $50(50)$ \\
Accuracy [TP + TN $) /$ total No of patients] & $82(86)$ & $87(87)$ \\
Predictive value: & $93(93)$ & $85(85)$ \\
"If present" [TP/(TP + FP)] & $57(71)$ & $100(100)$ \\
"If absent" [TN/(TN + FN)]
\end{tabular}

*"One look," "two looks" refer to all 22 patients whose urine was examined once and to the 15 whose urine was examined for a second time during one day renal function testing. Abnormality present at either examination was taken as positive result; no abnormality detected at either examination was taken as negative result. +Figures in parentheses are values obtained when only two out of three false negatives were accepted as truly false negative (see table II).

TABLE II-Raw data for calculations in table I

\begin{tabular}{lccccc}
\hline & $\begin{array}{c}\text { True } \\
\text { positives } \\
\text { (TP) }\end{array}$ & $\begin{array}{c}\text { False } \\
\text { positives } \\
\text { (FP) }\end{array}$ & $\begin{array}{c}\text { True } \\
\text { negatives } \\
\text { (TN) }\end{array}$ & $\begin{array}{c}\text { False } \\
\text { negatives } \\
\text { (FN) }\end{array}$ & $\begin{array}{c}\text { Total } \\
\text { No of } \\
\text { patients }\end{array}$ \\
\hline "One look" & 14 & 1 & 4 & 3 & 22 \\
"Two looks" & 11 & 2 & 2 & 0 & 15 \\
\hline
\end{tabular}

Twenty four hour urinary protein excretion was measured by routine turbidometric techniques by the hospital's biochemistry laboratory and graded for comparison with cast excretion as : normal = $0-200 \mathrm{mg}, 1=200-3500 \mathrm{mg}, 2=3 \cdot 5-10 \cdot 0 \mathrm{~g}, 3=10-20 \mathrm{~g}$, and $4=-20 \mathrm{~g} /$ day.

Sensitivity was calculated as the number of true positives (patients with both histological findings and numbers of casts abnormal) divided by the number of patients with the disease (true positives plus false negatives); specificity as the number of true negatives divided by the number of patients without disease (true negatives plus false positives); accuracy as the number of true positives plus true negatives divided by the total number of patients; positive predictive value ("if present," patient has histological abnormality) as the number of true positives divided by the number of patients with abnormality (true positive plus false positive); and negative predictive value ("if absent," patient has no histological abnormality) as the number of true negatives divided by the number of patients without the abnormality (true negative plus false negative).

Renal function was considered to be abnormal if true endogenous creatinine clearance (glomerular filtration rate) corrected for age was $<82 \%$; if maximum urinary osmolality/plasma osmolality -1 after vasopressin in oil was less than $-2 \mathrm{SD}$ of that predicted for the patient's urine flow corrected to $1 \mathrm{dl}$ glomerular filtration rate; or if minimum urinary $\mathrm{pH}$ was $>5.2$ after an acid load and blood gas control. ${ }^{5}$

\section{Results}

Outcomes of the two histological examinations in each case (those performed blindly and by the routine laboratory) showed no appreciable differences. In five of the 22 patients the histological findings were normal. Of the remainder, however, eight showed minimal, one mild, six moderate, and two severe changes. The histological changes together with the clinical picture suggested IgA disease in five cases, lupus nephritis in three, chronic glomerulonephritis in three, diabetes in one, chronic pyelonephritis in one, probable lithium nephropathy in one, and minimal lesion nephrotic syndrome in one; two patients were unclassifiable.

TABLE II-Correlation of presence or absence of renal disease with urine microscopy and renal functional abnormalities. (Figures in parentheses are values for "two look" specimens)

Abnormalities tested

\begin{tabular}{|c|c|c|c|c|c|c|}
\hline & Casts & $\begin{array}{l}\text { Casts } \\
\text { (without steroid treatment) }\end{array}$ & Red blood cells & $\begin{array}{l}\text { Casts or } \\
\text { red blood cells }\end{array}$ & $\begin{array}{l}\text { Casts and } \\
\text { red blood cells }\end{array}$ & Renal function \\
\hline \multirow{2}{*}{$\begin{array}{l}\text { No of patients } \\
\text { Sensitivity }(\%) \\
\text { Specificity }(\%) \\
\text { Accuracy }(\%) \\
\text { Predictive value: } \\
\text { "If present" ( } \%) \\
\text { "If absent" }(\%)\end{array}$} & $\begin{array}{ll}22 & (15) \\
82(100) \\
80(50) \\
82(87)\end{array}$ & $\begin{array}{ll}19(13) \\
86(100) \\
80(50) \\
84(85)\end{array}$ & $\begin{array}{l}22(15) \\
53(73) \\
60(50) \\
55(67)\end{array}$ & $\begin{array}{l}22(15) \\
88(100) \\
40(25) \\
77(80)\end{array}$ & $\begin{array}{r}22(15) \\
41(73) \\
100(75) \\
55(73)\end{array}$ & $\begin{array}{r}15 \\
64 \\
100 \\
73\end{array}$ \\
\hline & $\begin{array}{l}93(85) \\
57(100)\end{array}$ & $\begin{array}{l}92(82) \\
67(100)\end{array}$ & $\begin{array}{l}82(80) \\
27(40)\end{array}$ & $\begin{array}{l}83(79) \\
50(100)\end{array}$ & $\begin{aligned} 100(89) \\
33(50)\end{aligned}$ & $\begin{array}{r}100 \\
50\end{array}$ \\
\hline
\end{tabular}

in small amounts, no crescents etc, tubules back to back with little apparent interstitium, occasional mononuclear cell present); mild $=2$ (average number of glomerular nuclei over 200 but not obviously increased, no segmental inflamed lesions, mesangium apparent in most or all glomeruli as a fine pink thread, series of three mesangial nuclei in a line, some increase in interstitium with slight separation of tubules and sparsely scattered mononuclear cells in the interstitium); moderate $=3$ (scattered segmental inflammatory lesions in some glomeruli (less than half) with mild or minimal crescents allowed, rare subendothelial deposits, interstitium showing definite areas (less than half) of increase (oedema or fibrosis) with sparse or focal dense mononuclear infiltration); severe $=4$ (more than half of the glomeruli with inflamed segments, and florid crescent formation in more than a quarter of the glomeruli, subendothelial deposits easy to find, frank interstitial increase with obvious interstitial infiltrate).

Fifteen of the patients also underwent one day renal function testing, ${ }^{5}$ in which urine specimens were examined by the same technique and the same person and gave what we refer to as "two look" specimens, because they provided a look at a second specimen from these patients. Although no special precaution was taken to code the specimens, the examiner was kept unaware of the histological diagnosis by the manner in which the urine specimens were examined.
Table I shows the percentage sensitivity, specificity, accuracy, and positive and negative predictive values of urine examination in relation to abnormal renal histological findings. Table II gives the raw data. For comparison, table III summarises the percentages calculated for the various urinary abnormalities. Looking at urinary casts only and comparing this with renal functional abnormalities it was apparent that the latter were less sensitive and accurate but very specific; and "if present" it was $100 \%$ certain that renal histological findings would be abnormal. This was to be expected, since functional abnormalities (mainly in the glomerular filtration rate) are manifested relatively late in renal disease; hence testing renal function was not particularly sensitive in predicting histological abnormalities, and its negative predictive value was even worse. Casts alone were more sensitive and accurate, similar in negative predictive value, but less specific. Pooling the results of the "two look" urine examinations for casts improved nearly all indices except specificity.

Testing urine specimens from patients not taking steroids yielded no significant differences in values (table III). Taking red blood cells alone gave the worst results and was very non-specific but had a relatively high positive predictive rating. Combining casts with red cells generally did not give better results. Table III separates the results for urine specimens required to contain either abnormal red 
cells or casts and for specimens required to contain abnormal numbers of red cells and casts before being regarded as abnormal.

The figure shows the relation between histological grading and number of casts. Numbers increased significantly with severity of the histological abnormality (Spearman's correlation coefficient for "one look" urine specimens: $\left.r_{s}: 0.630 ; p<0.01\right)$.

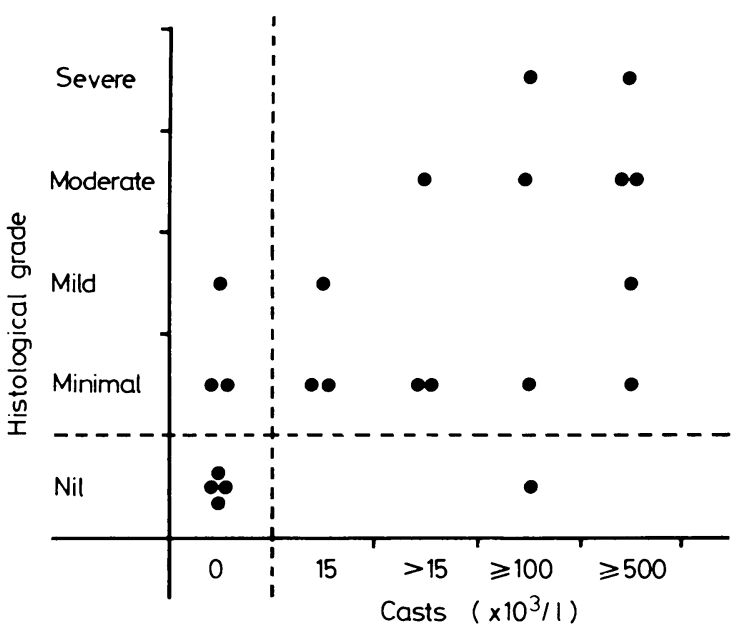

Relation between histological grading at light microscopy of biopsy specimens and number of casts excreted. Points are patients. (Spearman's correlation coefficient $0.630 ; \mathrm{p}<0.01$.)

Protein excretion correlated poorly with histological abnormality$r=0.318(p-0.10)$ for all patients and 0.314 when patients taking steroids were excluded. Proteinuria correlated slightly better with number of casts excreted, with $r$ values of $0.538(p<0.02)$ for all patients and $0.513(\mathrm{p}<0.05)$ when patients taking steroids were excluded.

\section{Discussion}

The quantitative method of urine microscopy employed in our series was a sensitive and reliable predictor of renal disease. Treatment with steroids in doses of $12 \cdot 5-60.0 \mathrm{mg}$ a day did not significantly interfere with the results. Using the presence of casts or red cells as a criterion of abnormality did not improve results, while using the presence of casts and red blood cells as a criterion worsened sensitivity and negative predictive value but improved specificity and positive predictive value. The latter figures were similar to those comparing renal function with histological findings, indicating that functional changes and possibly excretion of red blood cells occur relatively late in renal disease when histological evidence is certainly present; hence, although specific and with a high positive predictive value, they are less useful in predicting early renal disease. Although dysmorphic red cells have been shown to be associated with glomerular disease ${ }^{6}$ the sensitivity of abnormal red cell numbers or types in predicting the presence or absence of renal disease has never been studied quantitatively.

In our study there were three false negative findings and one false positive (table II). The latter patient, a 33 year old man, had only hyaline casts without orthostatic proteinuria, confirmed in a second specimen. We cannot exclude the possibility that hyaline casts may not be associated with disease, but in a series of 1720 normal urine samples, only seven were found to contain these casts in abnormal numbers. ${ }^{4}$ The patient did have a history of recurrent frequency and haematuria and the biopsy specimen may not have picked up medullary changes. Of the three patients with false negative findings, two had only minimal changes. One, a 45 year old woman, had only one abnormal glomerulus, which may well have represented early changes with age rather than disease. She also gave a history of recurrent infections with increased white and red cells in the urine which may well have been from the lower urinary tract. She was receiving $12.5 \mathrm{mg}$ prednisolone daily for clinical systemic lupus erythematosus. We did not consider this patient to be genuinely false negative but a true negative; we therefore recalculated our results to allow for this patient and found a sensitivity of $88 \%$, a specificity of $83 \%$, an accuracy of $86 \%$, a positive predictive value of $93 \%$, and a negative predictive value of $71 \%$ for the method (table I). The second patient, also with clinical systemic lupus erythematosus, was a 37 year old woman, not receiving steroids, who had casts in the second urine specimen; while the third patient, with mild changes, was a 48 year old man with a history of excess analgesic intake and who was also found to have casts on the second urine examination.

The relatively high correlation of excretion of casts with the severity of the histological changes was a surprise finding; casts usually fragment when they leave their site of formation and numbers counted may not represent numbers formed. Plainly, however, higher numbers of casts/ 1 of urine must reflect severity of the disease process and possibly indicate the greater number of nephrons affected.

Looking for casts in this manner is simple, taking six to seven minutes at the most, and is eminently suitable as routine practice to detect renal disease. The Addis count ${ }^{7}$ is less reliable, since it necessitates 12 or 24 hour collections and suffers from the likelihood that formed elements may disappear, especially when the samples are alkaline. ${ }^{8}$ The present method does not measure excretion rates, as does the Addis count, but does correspond to measured excretion rates if one assumes a daily urine volume of $1250 \mathrm{ml}$. Variation in daily urine volume probably explains the $5-13 \%$ of false positive and false negative results (these suggest variations in daily urine volumes of 1200-1400 ml/day, which seem reasonable).

Though the technique is simple, it has satisfactory sensitivity, specificity, accuracy, and positive predictive powers; sensitivity and negative predictive powers may be improved on if two early morning specimens are examined, although specificity then suffers. Since usually only one or at the most two specimens from a patient are examined before deciding on further action, it was not the aim of this study to examine the effects of reproducibility of the method on its predictive powers. Such a study would require multiple biopsies. From our results, however, it is clear that if no casts can be detected in two urine samples the likelihood of finding anything more than minimal changes on renal biopsy is very small, and biopsy would not need to be performed unless a clinical trial or an important change of treatment was in prospect. This latter is unlikely, since already minimal changes can be detected by this method which would not be expected to change any treatment not already contemplated clinically, such as in systemic lupus erythematosus. Avoiding biopsy is of course important, since about $80 \%$ of all biopsies result in significant perinephric haematomas, ${ }^{9}$ although actual loss of a kidney is rare.

We made no effort to correlate the types of casts excreted with the type of histological abnormality, clinical picture, or stage of the disease. Such a study would need to be much larger and be over a greater length of time, and we doubt that it would add appreciably to detecting the presence or absence of renal disease.

Microscopy of urine when performed properly is a highly reliable index of renal disease, and the disrepute that it has earned over the past few years is the result of very poor methodology, often explained by high workload. In the case of overuse of pathology services urine examinations for infection should be separated from those for renal disease and the latter regarded and performed with the seriousness and dedication usually reserved for blood film examinations. If this cannot be done by a laboratory, then in view of the high rate of false negative results with inadequate techniques ${ }^{2}$ probably such urine examinations should not be done at all. 
' Kesson AM, Talbot JM, Györy AZ. Microscopic examination of urine. Lancet 1978 ;ii :809-12.

Mahajan SK, Ordonex NG, Feitelson PJ, et al. Lupus nephropathy without clinical renal involvement. Medicine (Baltimore) 1977;56: 493-501.

${ }^{3}$ Appel GB, Silva FG, Pirani CL, et al. Renal involvement in systemic lupus erythematosus (SLE). Medicine (Baltimore) 1978;57:371-410.

4 Györy AZ, Kesson AM, Talbot JM. Microscopy of urine-now you see it, now you don't! Am Heart $\mathcal{F}$ 1980;99:537-8.

${ }^{5}$ Györy AZ, Edwards KDG, Stewart JH, et al. Comprehensive one-day renal function testing in man. $\mathcal{f}$ Clin Pathol 1974;27:382-91.
${ }^{6}$ Fairley KF, Birch DF. Hematuria: a simple method for identifying glomerular bleeding. Kidney Int 1982;21:105-8.

${ }^{7}$ Addis $T$. The number of formed elements in the urinary sediment of normal individuals. 7 Clin Invest 1926;2:409-15.

* Triger DR, Smith JWG. Survival of urinary leucocytes. $f$ Clin Pathol 1966;19:443-7.

${ }^{9}$ Rosenbaum R, Hoffsten PE, Stanley RJ, et al. Use of computerized tomography to diagnose complications of percutaneous renal biopsy. Kidney Int 1978;14:87-92.

(Accepted 21 December 1983)

\title{
Hypertrophic osteoarthropathy in cystic fibrosis
}

\author{
STANLEY BRAUDE， HARRY KENNEDY， MARGARET HODSON，JOHN BATTEN
}

\begin{abstract}
Seven adult patients with cystic fibrosis who had radiological evidence of hypertrophic osteoarthropathy were reviewed. In five of the patients symptoms were particularly pronounced at times of acute infective exacerbations; appropriate treatment of the infective episodes resulted in reduction or resolution of the bone pain and joint effusions. Despite this symptomatic relief periosteal changes persisted radiologically and their chronic nature was indicated by changes in the midshafts of long bones. Four of the seven patients had transient gynaecomastia or mastalgia related to infective exacerbations.

It is hypothesised that a neuroendocrine mechanismnamely, release of vasoactive intestinal polypeptidemight account for the osteoarthropathy.
\end{abstract}

\section{Introduction}

Hypertrophic osteoarthropathy is said to be rare in cystic fibrosis and has a variable relation to the severity of the underlying pulmonary disease..$^{1-3}$ We report on seven patients with cystic fibrosis who had radiologically proved hypertrophic osteoarthropathy. Although gynaecomastia and hypertrophic osteoarthropathy are well recognised in squamous bronchogenic carcinoma, we do not think that they have been reported previously in association with cystic fibrosis.

\section{Details of patients}

Seven patients (six men, one woman) with cystic fibrosis were investigated radiologically because of bone pain and were found to have hypertrophic osteoarthropathy. The table gives details of clinical and laboratory features. We report on one of the patients (case 1) in greater detail below.

Case report-A 21 year old man known to have had cystic fibrosis for 15 years was admitted with a two week history of increasing dyspnoea and production of sputum. Over the week before admission

Brompton Hospital and Cardiothoracic Institute, University of London

STANLEY BRAUDE, MRCP, registrar in thoracic medicine

HARRY KENNEDY, MRCP, senior house officer in thoracic medicine

MARGARET HODSON, MD, FRCP, senior lecturer in thoracic medicine and honorary consultant physician

JOHN BATTEN, MD, FRCP, consultant physician

Correspondence to: Dr S Braude, Lower Medical Corridor, Hammersmith Hospital, London W12 0HS.

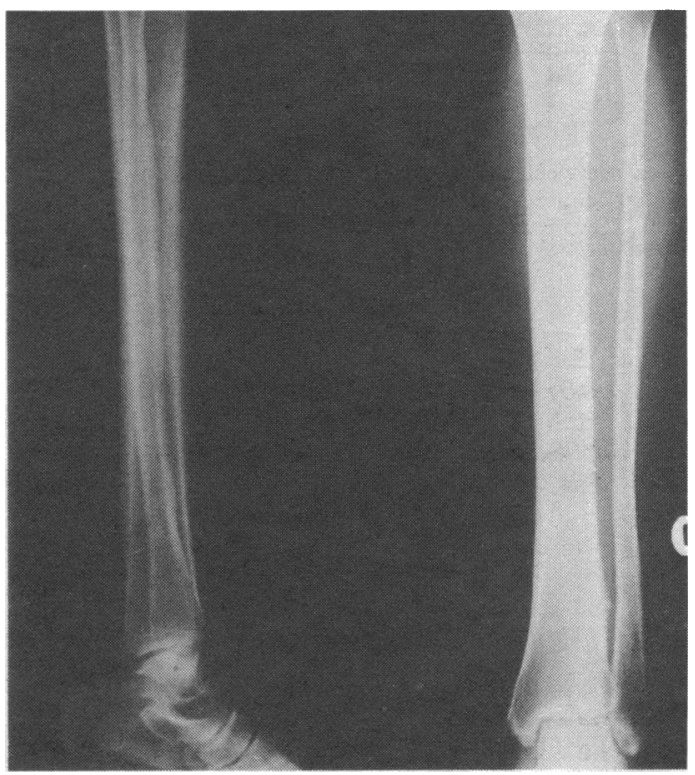

FIG 1 -New bone seen in midshaft of fibula with no intervening band of radiolucence.

he had noted that his ankles and knees had become swollen and tender. He had a history of similar joint symptoms associated with exacerbation of his chest disease. On examination he had gross finger clubbing with swollen, hot ankle joints bilaterally and a left knee effusion. The wrists were not swollen, and all joint movements were full. Bilateral gynaecomastia was present. Clinical signs in the chest were unchanged from those at previous examinations, but there was clear evidence of physiological deterioration (forced expiratory volume in one second and vital capacity 800 and $1400 \mathrm{ml}$ compared with 1250 and $1950 \mathrm{ml}$ three months previously). Sputum culture grew mucoid, fully sensitive Pseudomonas aeruginosa, and he was treated with intravenous piperacillin and gentamicin. Radiographs of both tibias showed periosteal reactions. With treatment of his pulmonary disease the arthropathy and gynaecomastia resolved completely within 10 days, but radiological follow up two months after discharge showed that the periosteal changes persisted.

All seven patients had the characteristic radiological appearance of hypertrophic osteoarthropathy, with periosteal reaction separated from underlying cortex by a thin radiolucent line at the ends of long bones. In addition, all patients showed a characteristic abnormality of the midshaft to some extent: new bone was seen apparently fused with the cortex of the shaft with no intermediate band of radiolucency (fig 1). This has previously been described as a sign of the chronic nature of the periostitic process. ${ }^{4}$ In one patient the distribution of periosteal reaction was unusual, the proximal femur being affected (fig 2). No 